of Fizeau's water-tube experiment, the prediction of the law connecting electronic mass with velocity, and the prediction of ponderomotive electromagnetic forces in moving media.

One final, and therefore crucial, test remains : gravitation. It was soon noticed that the hypothesis was inconsistent with the exact truth of Newton's gravitational law of force $m m^{\prime} / r^{2}$. Thus the hypothesis of relativity predicts that a freely moving planet cannot describe a perfect ellipse about the sun as focus. This prediction is made on quite general grounds, just as the conservation of energy predicts that a stream of water cannot flow uphill. But the conservation of energy by itself is powerless to predict what will be the actual course of a stream of water, and in precisely the same way the hypothesis of relativity alone is powerless to predict what will be the orbit of a planet. Before this or any other positive gravitational predictions can be made, additional hypotheses must be introduced. The main trunk of the tree is the relativity hypothesis already mentioned; these additional hypotheses form the branches. The trunk can exist without its branches, but not the branches without the trunk. Whether the branches have been placed on the trunk with complete accuracy is admittedly still an open question-it must of necessity remain so until the difficult questions associated with the gravitational shift of spectral lines have been finally settled-but the main trunk of the tree can be disturbed by nothing short of a direct experimental determination of the absolute velocity of the earth, and the only means which can possibly remain available for such a determination now are gravitational.

\title{
The Michelson-Morley Experiment and the Dimensions of Moving Bodies.
}

\section{By Prof. H. A. Lorentz, For.Mem.R.S.}

A doubts have sometimes been expressed concerning the interpretation of Prof. Michelson's celebrated experiment, some remarks on the subject will perhaps not be out of place here. I shall try to show, by what seems to me an unimpeachable mode of reasoning, that, if we adopt Fresnel's theory of a stationary æther, supposing also that a material system can have a uniform translation with constant velocity $v$ without changing its dimensions, we must surely expect the result that was predicted by Maxwell.

Let us introduce a system of rectangular axes of co-ordinates fixed to the material system, the axis of $x$ being in the direction of the motion. Then, with respect to these axes, the æther will flow with the velocity $-v$. The progress of waves of light, relatively to them, may be traced by means of Huygens's principle; for this purpose it suffices to know the form and position of the elementary waves. For the sake of generality I shall suppose the propagation to take place in a material medium of refractive index $\mu$, so that, if $c$ is the velocity of light in the æther, the velocity in the medium when at rest would be $c / \mu$. The elementary wave formed in the time $d t$ around a point $\mathrm{P}$ will be a sphere of radius $(c / \mu) d t$, of which the centre $\mathrm{P}^{\prime}$ does not, however, coincide with $\mathrm{P}$, the line $\mathrm{PP}^{\prime}$ being in the direction opposite to that of $\mathrm{OX}$, and having the length $\left(v / \mu^{2}\right) d t$ (Fresnel's coefficient).

If $Q$ is any point on the surface of the sphere, $P Q$ can be considered as an element of a ray of light, and $w=\mathrm{PQ} / d t$ will be the velocity of the ray. Confining ourselves to terms of the second order, i.e. of the order $v^{2} / c^{2}$, and denoting by $\delta$ the angle between the ray and $\mathrm{OX}$, we have

$$
\frac{\mathrm{I}}{w}=\frac{\mu}{c}+\frac{v}{c^{2}} \cos \delta+\frac{v^{2}}{2 \mu c^{3}}\left(\mathrm{I}+\cos ^{2} \delta\right) .
$$

Now, let $\mathrm{A}$ and $\mathrm{B}$ be points having fixed positions in the material system. The course $s$ of a ray of light passing from $A$ to $B$ will be determined by the condition that the integral

$$
\int \frac{d s}{w}
$$

is a minimum. Using the above value of $1 / w$, it is easily shown that, if quantities of the second order are neglected, the course of the ray is not affected by the translation $v$, so that, if $L_{0}$ is the path of the ray in the case $v=0$, and $L$ the real path, these lines will be distant from each other to an amount of the second order only. Hence, if in the case of a translation $v$ we calculate by means of ( 1 ) the integral (2), both for $L$ and $L_{0}$, the two values will differ by no more than a quantity of the fourth order; indeed, since the integral is a minimum for $\mathrm{L}$, the difference must be of the second order with respect to the distances between $L$ and $L_{0}$, and these distances are already of the second order of magnitude.

It is seen in this way that, so long as we neglect terms of an order higher than the second, we may replace

$$
\int_{i} \frac{d s}{w} \text { by } \int_{L_{0}} \frac{d s}{w},
$$

an argument that must not be overlooked in the theory of the experiment. On the ground of it we shall commit no error if, in determining the paths $L_{1}$ and $L_{2}$ of two rays that start from a point $A$, and are made to interfere at a point $B$, we take no account of the motion of the apparatus. The change in the difference of phase produced by the translation will be given by the difference between the values which the integral

$$
\int \frac{z^{2}}{2 \mu c^{3}}\left(\mathrm{I}+\cos ^{2} \delta\right) d s
$$

takes for the lines $L_{1}$ and $L_{2}$ so determined. If, along the first of them, $\cos ^{2} \delta=1$, and along the 
second $\cos ^{2} \delta=0$, and $\mu=\mathrm{I}$, the change will be the same as would be produced by a lengthening of $L_{1}$ in the ratio of $\mathrm{I}$ to $\mathrm{I}+v^{2} / 2 c^{2}$. As no displacement of the fringes has been observed, we are led to the well-known hypothesis of a contraction of moving bodies in the direction of translation, in the ratio of $I$ to $I-v^{2} / 2 c^{2}$.

We could now try to extend the above considerations to cases in which $v / c$, though always below I, is no longer a small fraction. This would require somewhat lengthy calculations, into which, however, we need not enter here, because we know by the theory of relativity that the true value of the coefficient of contraction is $\sqrt{x-v^{2} / 2 c^{2}}$. I may remark here that there can be no question about the reality of this change of length. Suppose that, in studying the phenomena, we use a system of rectangular co-ordinates $x_{1}, x_{2}, x_{3}$, and a time $t$, and that in this system the velocity of light is $c$ in all directions. Further, let there be two rods, I. and II., exactly equal to each other, and both placed in the direction of $x_{1}$, I. at rest in the system of co-ordinates, and II. moving in the direction of its length with a velocity $v$. Then, certainly, if the length of a rod is measured by the differences of the values which the coordinate $x_{1}$ has at the two ends at one and the same instant $t$, II. will be shorter than I., just as it would be if it were kept at a lower temperature. I need scarcely add that if, by the ordinary transformation of the theory of relativity, we pass to new co-ordinates $x_{1}^{\prime}, x_{2}^{\prime}, x_{3}^{\prime}, t^{\prime}$ in such a manner that in this system the rod II. is at rest, and if now we measure the lengths by the difference between the values of $x_{1}^{\prime}$ which correspond to a definite value of $t^{\prime}$, I. will be found to be the shorter of the two.

The question arises as to how far the dimensions of a solid body will be changed when its parts have unequal velocities, when, for example, it has a rotation about a fixed axis. It is clear that in such a case the different parts of the body will, by their interaction, hinder each other in their tendency to contract to the amount determined by $\sqrt{I-v^{2} / c^{2}}$. The problem can be solved by the ordinary theory of elasticity, provided only that this theory be first adapted to the principle of relativity. Indeed, we can still use Hamilton's principle :-

$$
\delta \int_{t_{1}}^{t_{2}} d t \int(\mathrm{T}-\mathrm{U}) d \mathrm{~S}=0 .
$$

( $d \mathrm{~S}$, element of volume; $\mathrm{T}$, kinetic, and $\mathrm{U}$, potential, energy, both per unit of volume), if, by some slight modifications, the integral is made to be independent of the particular choice of co-ordin. ates. That this can be done, even in the general theory of relativity (theory of gravitation), is due to the possibility of expressing the length of a line-element in the four-dimensional space $x_{1}, x_{2}$, $x_{3}, x_{4}\left(x_{4}=t\right)$ in "natural units" $-i . e$. in such a manner that the number obtained for it is the same whatever be the co-ordinates chosen-and of measuring angles in a similar way. As is well

$$
\text { No. } 2677 \text {, VOL. IO6] }
$$

known, the length $d s$ of a line-element is given by the formula :-

$$
d s^{2}=\Sigma(a b) g_{a b} d x_{a} d x_{b}
$$

where the ten quantities $g_{a b}\left(g_{a b}=g_{b a}\right)$ are the gravitation potentials, and the angle $\delta$ between two elements is determined by

$$
\cos \delta d s d^{\prime} s=\Sigma(a b) g_{a b} d x_{a} d^{\prime} x_{b}
$$

In the sums, each of the indices $a$ and $b$ is to be given the values $I, 2,3,4$. When the value 4 is excluded, as will be the case in some of the following formulæ, a Greek letter will be used for the index.

We can also find an invariant value $l$ for the distance between two material particles $\mathrm{P}$ and $\mathrm{P}^{\prime}$ infinitely near each other. To this effect we have to consider the word-lines $\mathrm{L}$ and $\mathrm{L}^{\prime}$ of these particles in the space $x_{1}, x_{2}, x_{3}, x_{4}$. Let $Q$ be the point of $\mathrm{L}$ corresponding to the chosen time $x_{4}$, and $\mathrm{Q}^{\prime}$ a point of $\mathrm{L}^{\prime}$ such that $\mathrm{QQ}^{\prime}$ is at right angles to $L$. Then the length of $Q^{\prime}$, determined by means of (4), will be the value required. Similarly, if $\mathrm{P}^{\prime \prime}$ is a third particle, infinitely near $\mathrm{P}$ and $\mathrm{P}^{\prime}$, and $\mathrm{Q}^{\prime \prime}$ the point of its word-line so situated that $Q^{\prime \prime}$ is perpendicular to $\mathrm{L}$, the angle $\mathrm{P}^{\prime} \mathrm{PP}$ " will be taken to be the angle between the elements $Q Q^{\prime}$ and $Q Q^{\prime \prime}$ determined according to (5).

As to the co-ordinates $x_{1}, x_{2}, x_{3}, x_{4}$, it may be recalled that, in a field free from gravitation, they may be chosen in such a manner $\left(x_{1}, x_{2}, x_{3}\right.$ being at right angles to each other) that the velocity of light has the constant magnitude $c$; the potentials $g_{a b}$ will in this case have the values

$$
g_{11}=g_{22}=g_{33}=-\mathrm{I}, g_{44}=c^{2}, g_{a b}=0 \text { for } a \neq b \text {. }
$$

These may be called the normal values of the potentials, and a system of co-ordinates for which they hold a normal system.

Let us now consider a solid body $M$, and let us first conceive it to be placed in a normal system of co-ordinates $\left(S_{0}\right)$, and to be at rest in that system, free from all external forces. The body may then be said to be in its natural state, and its particles may be distinguished from each other by their co-ordinates $\xi, \eta, \xi$ with respect to three rectangular axes fixed in the body. In all that follows, these quantities will be constant, and so will be the mass $\rho d \xi d \eta d \zeta$ of an element, $\rho$ being the density in the natural state.

We shall now suppose the body to be placed in a system of co-ordinates $x_{1}, x_{2}, x_{3}, x_{4}(\mathrm{~S})$, not necessarily normal, and to have some kind of motion in that system. It is this motion, in which $x_{1}, x_{2}, x_{3}$ will be definite functions of $\xi, \eta, \zeta$, and $x_{4}$, which we want to determine by means of Hamilton's principle properly modified.

In order to get the new U, I shall introduce the dilatations $\xi_{\xi}, \eta_{\eta}, \zeta$, and shearing strains $\xi_{\eta}, \eta_{\zeta}, \zeta$, with respect to the axes $\xi, \eta, \zeta$. These quantities are defined as follows :-

Let $\mathrm{P}, \mathrm{P}^{\prime}$ be the particles $\xi, \eta, \xi$, and $\xi+d \xi, \eta, \zeta$, and let $l$ be their distance in the state considered 
(system S), and $l_{0}$ their distance in the natural state (system $\mathrm{S}_{0}$ ), these distances being both determined in the manner specified in what precedes. Then

$$
\xi \xi=\left(l-l_{0}\right) l_{l_{0}} .
$$

Again, if $\mathrm{P}^{\prime \prime}$ is the particle $\xi, \eta+d \eta, \xi$, and if the angle $\mathrm{P}^{\prime} \mathrm{PP} /$, calculated as stated before, has in the two cases the values $\delta$ and $\delta_{0}\left(=\frac{1}{2} \pi\right)$, we shall have

$$
\xi_{\eta}=\delta_{0}-\delta
$$

The six deformations $\xi_{\xi}$. . . will be considered as infinitely small. In the problem we have in view, they are of the order of magnitude $v^{2} / c^{2}$, so that our final result will be correct to that order.

If we put

$$
\begin{array}{r}
\mathrm{U}=\mathrm{A}\left(\xi \xi^{2}+\eta_{\eta}{ }^{2}+\zeta \xi^{2}\right)+\frac{1}{2} \mathrm{~B}\left(\xi_{\xi}+\eta_{\eta}+\zeta \xi^{2}+\right. \\
\frac{1}{2} \mathrm{~A}\left(\xi_{\eta}^{2}+\eta \zeta^{2}+\zeta \xi^{2}\right),
\end{array}
$$

the well-known expression for the potential energy of an isotropic elastic body, $U$ will be invariant for any change of co-ordinates.

As to the kinetic energy $\mathrm{T}$, it is to be replaced by an expression containing $\rho \frac{d s}{d t}$. Finally, we must write, instead of (3),

$$
\delta \int_{t_{1}}^{t_{2}} \int\left(-c \rho-\frac{\mathrm{I}}{c} \mathrm{U}\right) \frac{d s}{d t} d t d \xi d \eta d \zeta=0
$$

We have still to add the formulæ that are found by working out the above definitions of $\xi_{\xi}, \xi_{\eta}$, etc., viz. :--

$$
\begin{gathered}
\xi_{\xi}=-\frac{1}{2} \Sigma(a \beta) g_{a \beta} \frac{\partial x_{a}}{\partial \xi} \frac{\partial x_{\beta}}{\partial \xi}+\frac{\left[\Sigma(a \beta) g_{a \beta} v_{\beta} \frac{\partial x_{a}}{\partial \xi}\right]^{2}}{\Sigma \Sigma(a b) g_{a b} v a v_{b}}-\frac{1}{2}, \\
\xi_{\eta}=-\Sigma(\alpha \beta) g_{a \beta} \partial x_{a} \frac{\partial x_{\beta}}{\partial \xi}+\frac{\Sigma(a \beta) g_{a \beta} v_{\beta} \frac{\partial x_{a}}{\partial \xi} \cdot \Sigma(a \beta) g_{a \beta} v_{\beta} \frac{\partial x_{a}}{\partial \eta}}{\Sigma(a b) g_{a b} v a v_{b}}
\end{gathered}
$$

$\left\langle v_{1}, v_{2}, v_{3}\right.$ are the components of the velocity, and $v_{4}=\mathrm{I}$ ).

In our problem the body is supposed to move in a normal system of co-ordinates. By this our formulæ simplify to ${ }^{1}$

$$
\begin{gathered}
\delta \int_{t_{1}}^{t_{2}} \int\left(-c^{2} \rho-U\right)\left(1-\frac{v^{2}}{c^{2}}\right)^{\frac{1}{2}} d t d \xi d \eta d \zeta=0, \\
\xi_{\xi}=\frac{1}{2} \Sigma(a)\left(\frac{\partial x_{a}}{\partial \xi}\right)^{2}+\frac{\left[\Sigma(a) v_{a} \frac{\partial x_{a}}{\partial \xi}\right]^{2}}{2\left(c^{2}-v^{2}\right)}-\frac{1}{2},
\end{gathered}
$$

1 If in (7) we replace $\left(r-z^{2} / c^{2}\right)^{\frac{1}{4}}$ by $x-z^{2} / 2 c^{2}$, omitting the constant term $-c^{2} \rho$ and neglecting $U . z^{2} / 2 c^{2}$, we are led back to the ordinary formula (3).

$$
\xi_{\eta}=\Sigma(a) \frac{\partial x_{a}}{\partial \xi} \frac{\partial x_{a}}{\partial \eta}+\frac{\Sigma(a) v_{a} \frac{\partial x_{a}}{\partial \xi} \cdot \Sigma(a) v_{a} \frac{\partial x_{a}}{\partial \eta}}{c^{2}-v^{2}}
$$

When applied to a revolving body, these equations will enable us to determine the deformation that is produced, wholly independently of the theory of relativity, by centrifugal force, a deformation that will in reality far surpass the changes we want to consider. To get free from it we can consider the ideal case of a "rigid" body-i.e. a body for which the moduli of elasticity $A$ and $B$ in (6) are infinitely great. The centrifugal force will then have no effect on the dimensions, but the changes required by the theory of relativity will subsist. The assumption has also the advantage of simplifying the calculations; indeed, since $U$ becomes infinitely great, the term $-c^{2} \rho$ in (7) may be omitted.

I have worked out the case of a thin circular disc rotating with constant speed about an axis passing through its centre, at right angles to its plane. The result is that, if $v$ is the velocity at the rim, the radius will be shortened in the ratio of $\mathbf{x}$ to $\mathbf{I}-\frac{\mathbf{I}}{8} \frac{v^{2}}{c^{2}}$. The circumference changing to the same extent, its decrease is seen to be exactly one-fourth of that of a rod moving with the same velocity in the direction of its length. That there would be a smaller contraction was to be expected; indeed, the case can be compared to what takes place when a hot metal band is fitted tightly around a wheel and then left to cool.

At first sight our problem seems to lead to a paradox. Let there be two equal discs $A$ and $B$, mounted on the same axis, A revolving and B at rest. Then $\mathrm{A}$ will be smaller than $\mathrm{B}$, and it must certainly appear so (the discs. being supposed to be quite near each other) to any observer, whatever be the system of co-ordinates he chooses to use. However, we can introduce a system of co-ordinates $\mathrm{S}^{\prime}$ revolving with the disc $\mathrm{A}$; with respect to these it will be $B$ that rotates, and so one might think that now this latter disc would be the smaller of the two. The conclusion would be wrong because the system $S^{\prime}$ would not be a normal one. If we leave $\mathrm{S}$ for it, we must at the same time change the potentials $g_{a b}$, and if this is done the fundamental equation will certainly again lead to the result that $A$ is smaller than $B$.

\section{The Geometrisation of Physics, and its Supposed Basis on the Michelson-Morley Experiment.}

\section{By Sir Oliver Lodge, F.R.S.}

SO much has been written about the Michelson. $\checkmark$ Morley experiment that it would be needless to refer to it here, had it not been interpreted by philosophic writers in an interesting but overviolent ard, as some think, illegitimate manner. Historically it really does lie at the root of the remarkable attempt which is being made to geo- metrise physics, and to reduce sensible things like weight and inertia to a modification of space and time. The work of great Geometers has been pressed into the service, and a differentialinvariant scheme of expression has been utilised to do for physics in general, and especially for gravitation, what Maxwell's equations did for No. 2677 , VOL. IO6] 